\title{
Development of Method for Visualizing Behavioral States of Teams
}

\author{
Sixiong PENG ${ }^{\mathrm{a}, \mathrm{b}}$, Takashi AMAKASU ${ }^{\mathrm{a}, \mathrm{b}}$, Hiroki KAWAUCHI ${ }^{\mathrm{a}, \mathrm{b}}$, Hideyuki HORII ${ }^{\mathrm{b}}$ \\ and Kazuo HIEKATA ${ }^{\mathrm{a}}$ \\ ${ }^{\text {a } U n i v e r s i t y ~ o f ~ T o k y o, ~ J a p a n ~}$ \\ ${ }^{\mathrm{b}}$ Japan Social Innovation Center, Japan
}

\begin{abstract}
Visualizing behavioral states of teams is useful when providing effective reflection on teamwork for team members, monitoring and intervening teams, and analyzing team processes for teamwork researchers. This paper aims to develop a method to visualize behavioral states in meeting settings. We assumed that team processes follow several periods where behavioral states are stable and tried to detect these periods by focusing on change of communication patterns and facial expression. Detailed methods of detection and subsequent visualization are provided. We then test our method by comparing visualized team states in an idea generation workshop with qualitative observation of its team process. The result shows our method can effectively express some team states and provides viewpoints for more rigid quantitative verification.
\end{abstract}

Keywords. Teamwork, visualization, behavioral state, workshop

\section{Introduction}

Increasing number of transdisciplinary teams have been formed to address complex socio-technical issues nowadays. Although transdisciplinary teams have the potential to bring different skills and knowledge and create innovative solutions for complex issues, such teams are more prone to collapse without sufficient support for teamwork [1]. Inefficient teamwork could demotivate members and prevents information sharing, resulting in deterioration of efficiency, creativity, and resilience of the system.

Visualization of teamwork draws attention as a method for understanding current teamwork states and improving it. With a trend of advancement of information technology and online collaboration, various types of data about team interaction are accumulated such as text data in Social Networking Service and audiovisual data in virtual meetings. There are mainly two levels of focus; macro-level and meso-level visualization. Macro-level visualization deals with an interaction within a large organization or community consisting of several small groups or individuals. This type of research usually analyzes communication for a longer term, typically for several weeks or months. [2] used Sociometric Badges to collect data of the social network of workers at a call center of a large bank, and drew implication that was demonstrated to increase productivity. [3] developed software for visualizing communication flow and showed differences in communication patterns and network structures among three global communities on technology development. On the other hand, meso-level visualization deals with real-time interaction within a small group for a shorter term. 
Researchers have used nonverbal data to predict various constructs such as addressing [4], floor control [5], interest [6], team roles [7] and influence [8]. Given strong predictive power of nonverbal cues, visualizing and feedbacking these cues is proposed for manipulating teamwork. [9] developed "Second Messenger" which visualizes balance of speaking time among members and tested its usefulness in group decision making. [10] developed "Meeting Mediator" which can show interactivity of teams and participation of each member in real time based on data in sociometric badges. Although both of these projects prove the usefulness of the visualization for improving the member's behavior, it is the individual state that is visualized, not the team state that emerges from interaction of individuals and has strong relationship with team effectiveness [11]. By visualizing team states, members can stand on team's perspectives and consider a variety of ways to improve team states, not just thinking about increasing or decreasing the amount of speaking as in the previous visualization methods.

Thus, this paper attempts to visualize team states, particularly focusing on communication patterns. A communication pattern denotes the amount of interaction between every combination of group members. It can intuitively show participation of each member and indicators predicting performance of teams such as variance in the number of speaking turns [12] and centralization [13]. We present a method for visualizing a time-to-time change of communication pattern in a remote meeting setting, and demonstrate its usefulness in understanding and improving teamwork.

We focus on remote meetings because of two reasons. First, remote communication is rapidly becoming dominant with the outbreak of COVID-19 in 2020, leading to a drastic change in teamwork in any types of organizations. Decrease in face-to-face communication makes remote meetings an important place where team members integrate their knowledge and create values. Second, data in remote meetings provide audiovisual data of better quality for analysis. It contains less noise and can be easily separated by speakers because each speaker uses different microphones on their own computer. Besides, meetings can be recorded without preparing and setting recorders. Thus, supporting tools adjusted to remote settings can be easily applied to the real world.

\section{Method for Visualization}

\subsection{Data collection}

In order to calculate a communication pattern, we need speaking data including start time, end time, and index of a speaker for each utterance. For practicality, it is preferable to use a prevailing virtual communication tool to acquire data. Among widely-available tools, "ZOOM" would be suitable because it can allow users to record audio data of each account separately. Thus, this paper utilizes speaking data extracted from recording of ZOOM although our method can be applied to data from any other sources involving information described above.

\subsection{Detecting stable section of a communication pattern}

The simplest way to visualize communication patterns is to calculate a pattern every five minutes and to arrange it along a timeline. However, communication patterns change depending on the time span, which should be carefully considered upon visualization. We assumed that discussion is composed of multiple sections where communication 
patterns are relatively stable for more than five minutes, and decided to detect borders of these time spans. Five minutes are considered as a minimum length of time where communication patterns indicate a team state. To this end, we define degree of change (DC) as cosine similarity between a speaker change vector of the previous five minutes and the next five minutes. The speaker change vector consists of the number of turntaking between every combination of members as its element.

$$
D C(t)=1-\left(\begin{array}{c}
x_{11}^{t-5} \\
\vdots \\
x_{i j}^{t-5} \\
\vdots \\
x_{n n}^{t-5}
\end{array}\right) \cdot\left(\begin{array}{c}
x_{11}^{t} \\
\vdots \\
x_{i j}^{t} \\
\vdots \\
x_{n n}^{t}
\end{array}\right) \quad(t \geq 5)
$$

$x_{i j}^{t}$ : number of turn-taking between speaker $\mathrm{i}$ and speaker $\mathrm{j}$ within $\mathrm{t}$ to $\mathrm{t}+5$ minutes

We firstly calculate DC every thirty seconds. Then we calculate "peak" which satisfies the following conditions; the DC at the "peak" is maximal, its value is over 0.3 , and between neighboring "peaks" is at least one minimal DC which is smaller than the DC at the "peak" by at least 0.3 . Figure 1 shows the DC (blue line) along the time and "peak" (red dot) of our test data. "Peak" indicates the time point where the communication pattern of the previous five minutes and the next five minutes are highly different. Thus we use time of the "peak" as a border.

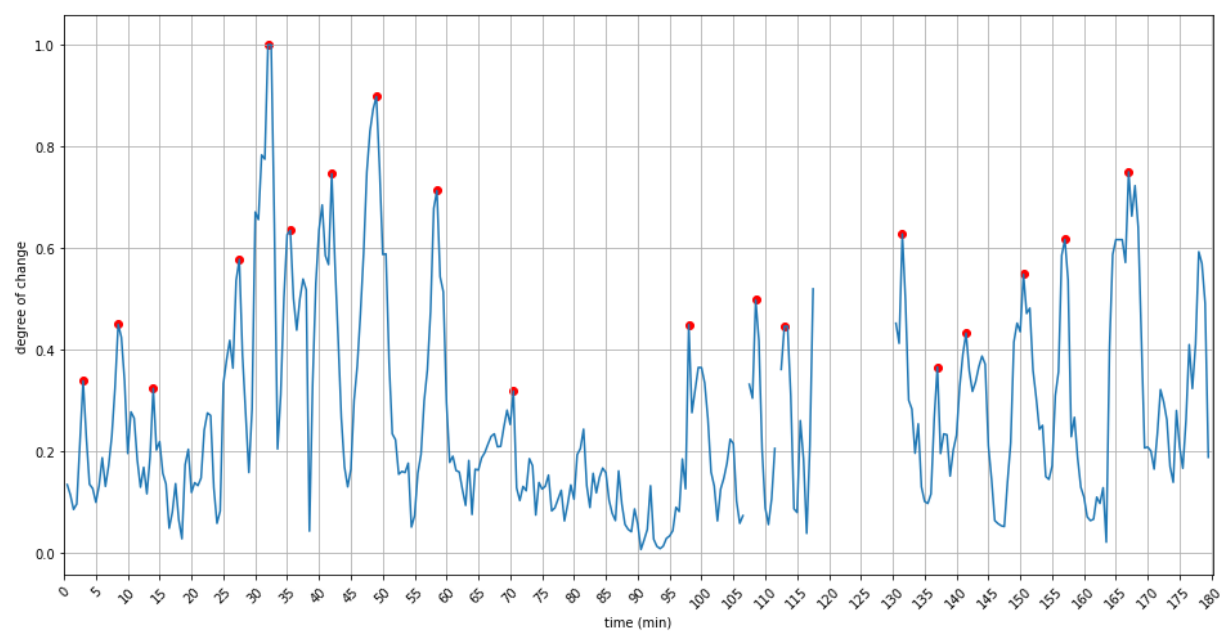

Figure 1. Degree of change and "peak" point of test data.

\subsection{Visualization with combining other features}

Finally, we calculate communication patterns in time spans between borders. To supplement information relating to team states, we additionally calculate coefficient of variance of the number of turn-taking between members (CV), the number of turn-taking per one minute (NUM), and speaking time of each member, and express them in the 
graph as Figure 2. Horizontal axis is time, vertical axis is the CV, the line width correlates with NUM. The diagram above the line shows the communication pattern, where each member is assigned to each vertex and the width of lines indicates the normalized number of turn-taking between two members and the size of circles at the vertex indicates the rate of speaking time. All of these features are calculated in time spans between borders. If the duration of the time span is shorter than five minutes, we change the color of the line and communication pattern to gray because it does not express team states in our definition. We additionally calculate a communication pattern during the whole period and display it in upper right. We have adopted a method of displaying these indicators that we thought is most intuitive and easy for viewers to understand.

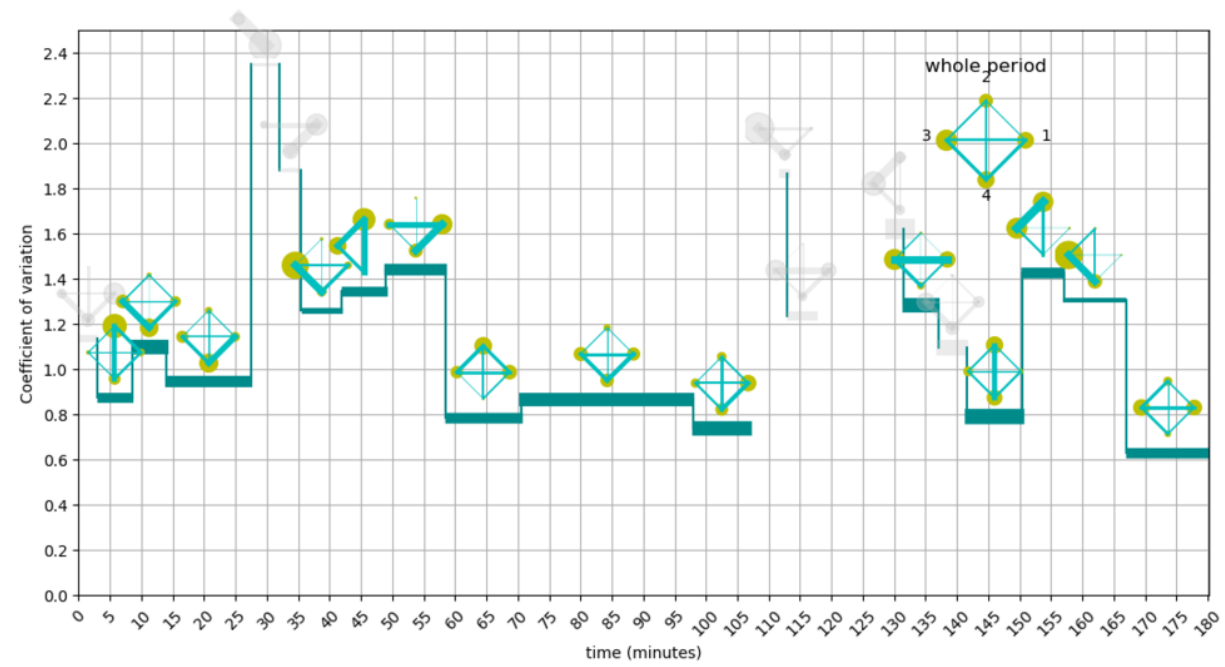

Figure 2. Visualization of teamwork in test data.

\section{Case study}

To demonstrate the usefulness of our visualization method, we applied it to remote discussions in an idea generation workshop.

\subsection{Workshop}

The "innovation in super-aged society" workshop was held in i.school, which is an innovation education program developed in University of Tokyo. This workshop aimed to create an idea to increase the number of "super-active senior people." The workshop consists of eight three-hour sessions and one eight-hour session within three months. All sessions in the workshop were conducted remotely using three virtual communication tools; ZOOM, Slack, and Apisnote. Six teams of five to six students joined the workshop. 
Participants include university students and company employees. Many of the participants were familiar with each other before the workshop started.

\subsection{Method}

As cases for analysis, it is suitable to select sessions with different communication patterns while controlling factors affecting communication patterns other than team states, so that the differences in communication patterns relating to team states would be clear. We choose the sessions of different groups on the same day in the early phase of the workshop. Because teams were instructed to generate ideas from scratch, the work were expected to be similar among all teams. We selected teams with six members, which are team A, C, D and F, considering its effect on communication patterns.

We examined the usefulness of the visualization method from two perspectives. First is within-session differences. In one session, the state of the team can change over time. For example, there can be times when only a few people are talking actively, and times when everyone is discussing equally. As within-session differences, we checked if our visualization can capture meaningful changes of team states in one session by qualitative comparison between visualized pictures and observation of video data. We particularly focused on the difference between sections with high and low CV or NUM. Another perspective is between-group differences. Overall team state can vary depending on sessions. Some teams may be in a positive mood and actively engage in discussion while anothers is not. we checked if our visualization can demonstrate differences of overall team state between sessions. Because our goal is to make hypotheses of what our method can indicate, rather than to test specific hypotheses, the investigation described above was conducted in an exploratory way. We listed insights from our visualization method, which will be tested in the future work.

\subsection{Result}

Figure3, Figure 4, Figure 5 and Figure 6 show the visualized teamwork of team A, C, D, and $\mathrm{F}$, respectively. In order to make them easier to analyze, the mean value of $\mathrm{CV}$ is indicated by a dotted line, and sections with higher NUM than its average among all sections are colored orange, and those with lower NUM are colored blue.

\subsubsection{Relationship between change of communication patterns and change of topics}

We compared visualized figures and observation of video data and found that most of the changes in communication patterns are associated with a change of topic of discussion. For example, Table1 shows discussion topics in the first one hour of team A. With a few exceptions (13:10 and 26:00), the communication pattern changes with the change of discussion topics.

\subsubsection{Negative correlation between $C V$ and NUM}

There is a common tendency that sections with higher NUM have lower CV. Negative correlation was found between these two values $(n=64, r=-.66, p=.0000)$. This could simply mean that sections with lower NUM didn't have enough opportunity for everyone to speak even if everyone had an equal probability to speak. Another interpretation would be that discussion by a limited number of members tends to be inactive in this workshop 


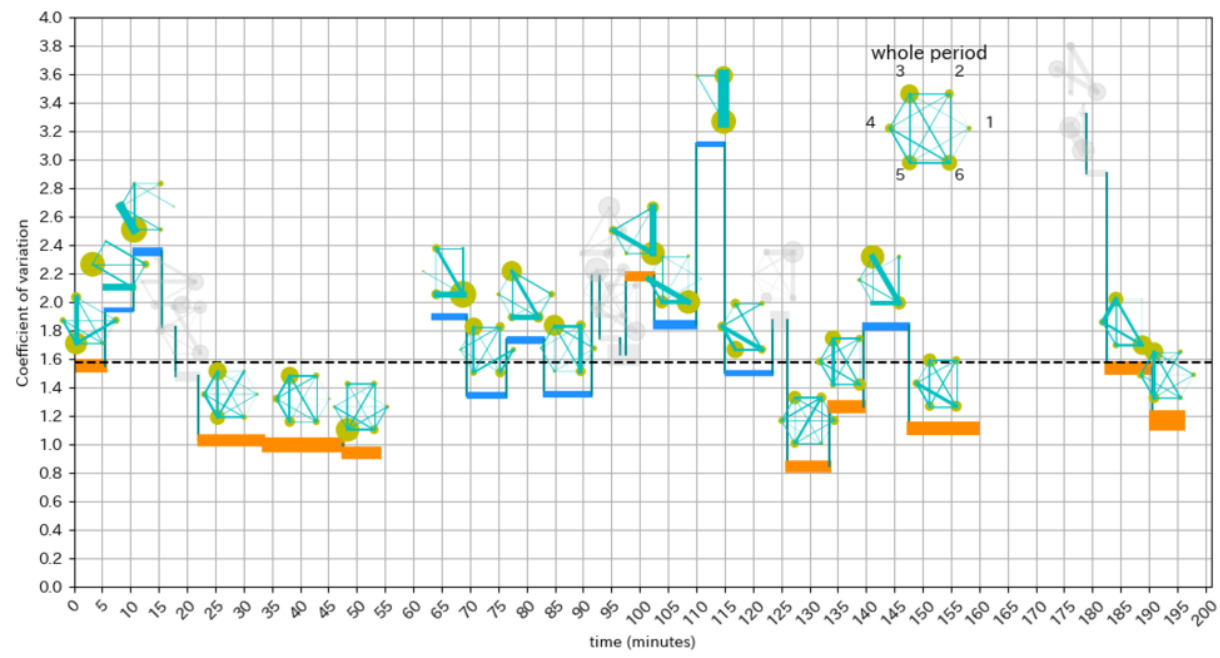

Figure 3. Visualization of teamwork of team A.

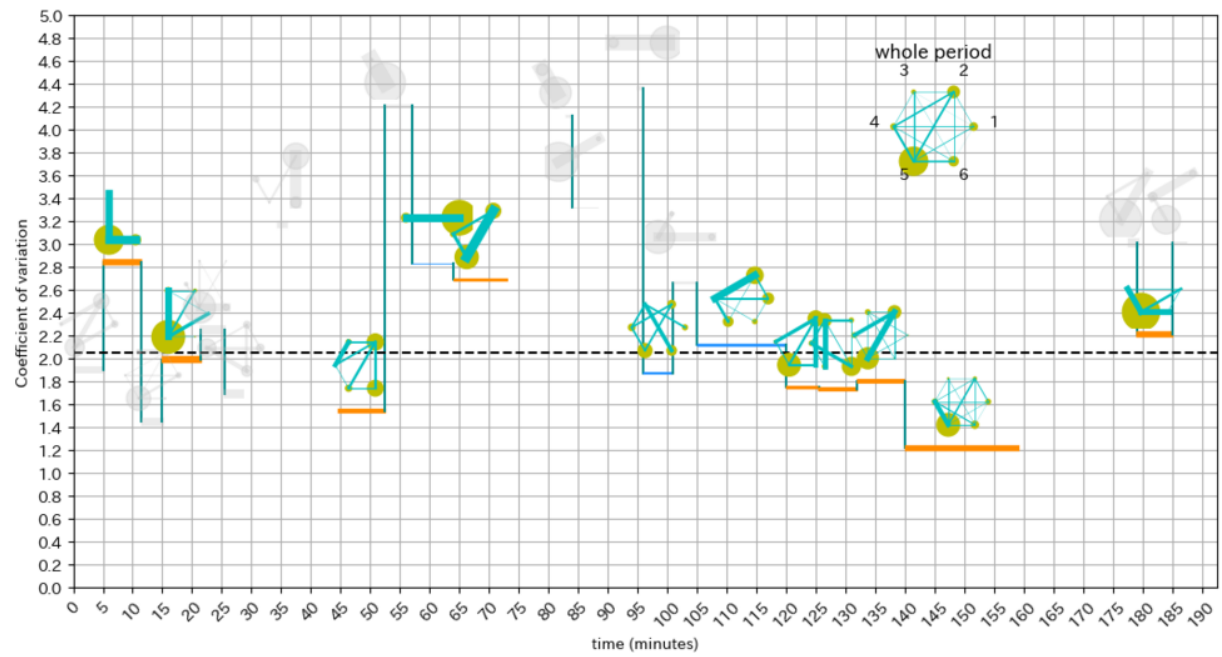

Figure 4. Visualization of teamwork of team C.

conditions. The latter interpretation could be true provided that the negative correlation was found even if CV and NUM are calculated for whole hours, where all participants have enough time to speak, with all sessions with six members $(\mathrm{n}=29, \mathrm{r}=-.51, \mathrm{p}=.0047)$. We further provided detailed observations of team states in sections with both higher and lower CV (lower and higher NUM, respectively) in the following part. 


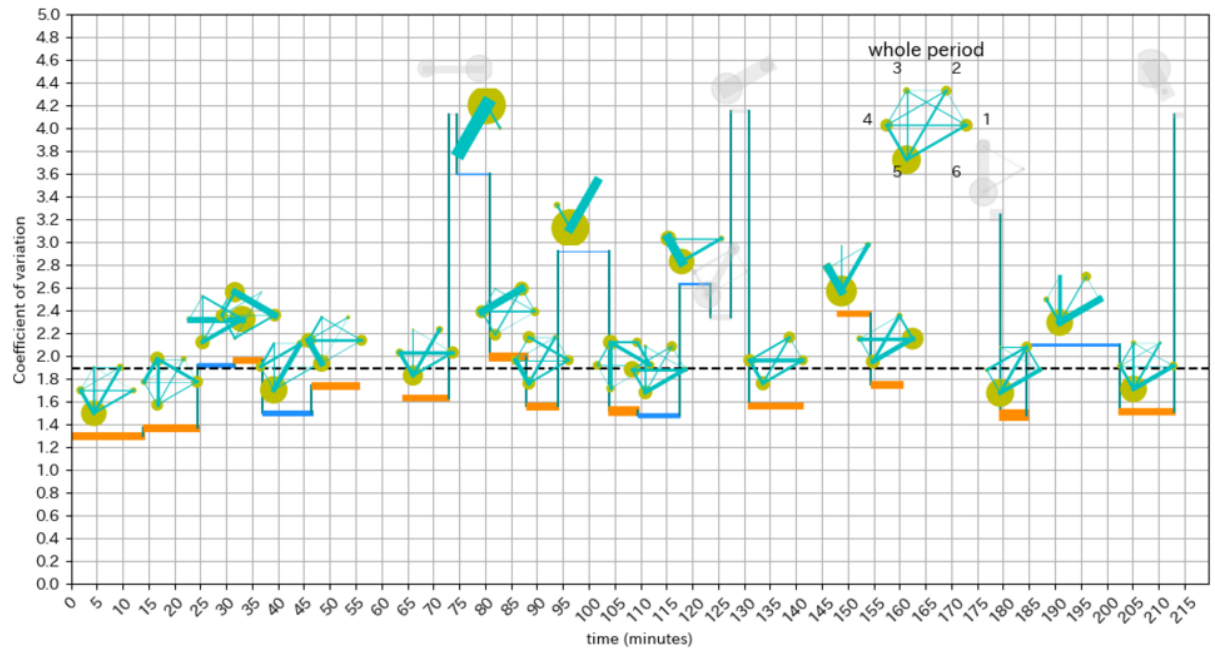

Figure 5. Visualization of teamwork of team D.

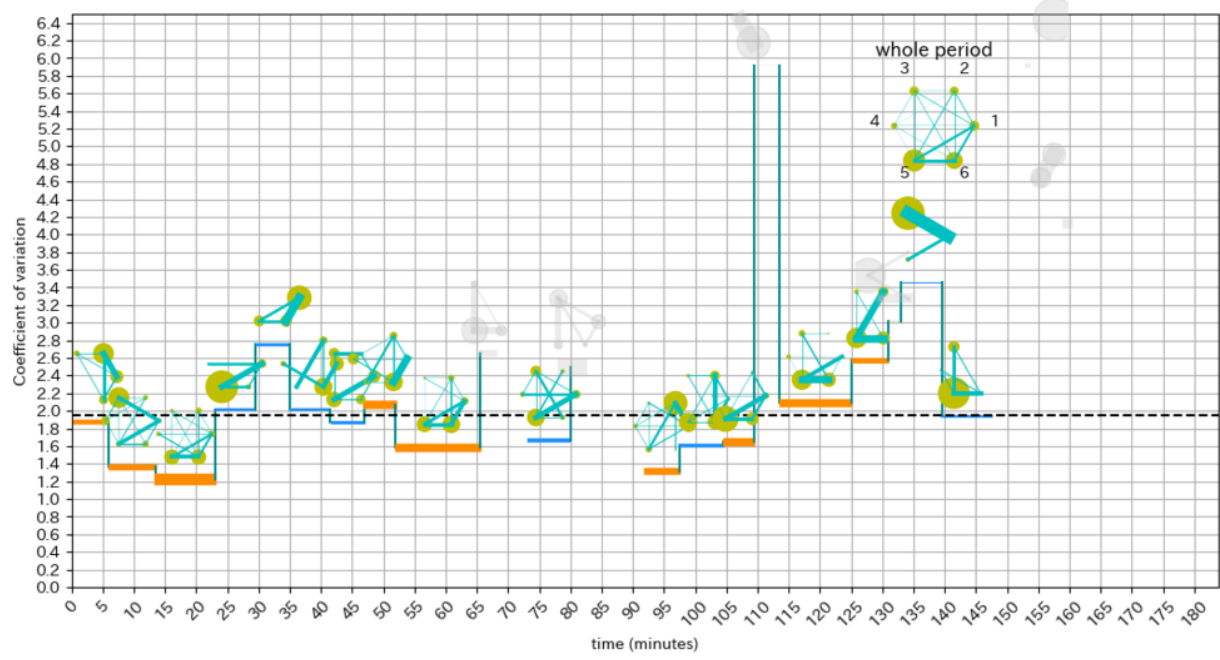

Figure 6. Visualization of teamwork of team F.

\subsubsection{Active discussion in a section with low $C V$ and high NUM}

Discussion seems to be more active in sections with low CV and high NUM. In these periods, members listen to each other and build on others' opinions, resulting in seamless discussion where topics were developed continuously. Even members with lower numbers of speaking seem to be concentrating on discussion. They show acceptance of others by smiling and nodding their head, creating a harmonious mood. Even when they 
Table 1. Discussion topics in the first one hour of team A.

\begin{tabular}{|c|c|}
\hline time & Discussion topic \\
\hline $0: 00$ & How to proceed today's discussion \\
\hline $5: 42$ & Definition of "super active senior" \\
\hline $13: 10$ & How to choose cases for analysis \\
\hline $21: 45$ & Categorizing cases \\
\hline $26: 00$ & Commonalities of cases \\
\hline $34: 46$ & Evaluation and selection of cases for analysis \\
\hline $48: 38$ & How to proceed the next discussion \\
\hline $51: 15$ & Confirmation of the reason for choosing the case \\
\hline
\end{tabular}

disagree with others and provide critical comments, their relationship would not be broken. These characteristics are more pronounced in a section with even lower CV and higher NUM such as a section from 22 to 54 minutes of team A.

\subsubsection{Shared understanding in a section with particularly lower CV and higher NUM}

Whether the team could build a shared understanding of the topics seems to differentiate sections with slightly lower CV than its average and sections with far lower CV. For example, both the discussion of team A from 22 to 54 minutes, and from 125 to 160 minutes were about evaluation of proposed ideas. Because ideas had been already shared among members, it was relatively easy for them to share their opinions. Hence, more members were able to actively participate in discussion, which resulted in lower CV and higher NUM. On the other hand, the discussion of team A from 0 to 5 minutes, and from 70 to 90 minutes were about sharing and elaborating new ideas. Because ideas were newly introduced, members had to simultaneously understand them and share their opinions, which resulted in relatively higher CV and the NUM. Another example is a discussion of team D from 15 minutes to 55 minutes. During this section, the team was discussing the criteria for evaluating ideas, but in the middle of the discussion, they started a conceptual discussion about the definition of "needs". This occurred from 14 to 37 minutes, when the CV was relatively higher. This indicates that in a conceptual discussion, it was difficult for members to understand others' opinions and create a shared understanding, resulting in decrease in the number of comments and participation.

\subsubsection{Several patterns of team states in period with high CV and low NUM}

As opposed to sections with low CV and high NUM, sections with high CV and low NUM contain several types of team states. Observed patterns were discussion about the next topic by limited members with higher engagement (team A from 10 to 15 minutes and from 98 to 110 minutes, team $\mathrm{D}$ from 148 to 155 minutes), discussion combined with a short individual work (team A from 64 to 70 minutes, team C from 68 to 73 minutes, team D from 73 to 81 minutes and from 94 to 103 minutes, team F from 23 to 28 minutes, 
Table 2. Mean CV, mean NUM, and the number of change of communication pattern in all teams.

\begin{tabular}{|c|c|c|c|}
\hline Team & Mean CV & Mean NUM & Number of changes in communication patterns \\
\hline A & 1.58 & 6.59 & 20 \\
\hline C & 2.05 & 2.81 & 12 \\
\hline $\mathrm{D}$ & 1.89 & 4.06 & 20 \\
\hline $\mathrm{F}$ & 1.95 & 3.44 & 17 \\
\hline
\end{tabular}

from 31 to 40 minutes, and 133 to 138 minutes), sharing information without discussion (team $\mathrm{C}$ from 56 to 73 minutes, team $\mathrm{F}$ from 125 to 131 minutes), and discussion facilitated by one member (team $\mathrm{C}$ from 5 to 11 minutes).

\subsubsection{Overall activity level}

Table. 2 shows mean CV and mean NUM of all sections and the number of change of communication patterns in all teams. Team A and team D show lower mean $\mathrm{CV}$, higher mean NUM, and more number of changes of communication patterns. We got an impression from the video data that discussion of these two teams are more active overall than the others in terms of fluency in discussion, higher concentration of members, and harmonious mood. Although the activity level of teams varies depending on the content of the discussion and the psychological state of the team members at the time, the baseline activity level can be assumed to be relatively stable, and the average value may represent it. We expect this value to be correlated with cohesiveness or other affective states measured by questionnaires in previous teamwork research.

\section{Discussion and Conclusion}

Although our result is based on qualitative observations of a limited number of cases, it provides some insights stimulating future research. Firstly, the possibility of our method of detecting active discussion is worth noticing, which is characterized by listening to and building on others' opinions, constant flow of discussion, concentration of all members, friendly mood, and critical comments while maintaining relationships. Previous research shows the importance of detecting such a moment. For example, group flow, which shares some traits with the active discussion, was considered as an optimal experience for a group where it can perform at its peak [14]. Empirical studies show its relationship with an increase of group efficacy [15] and an interest in discussion topics [16]. Other research on "hot spots," which are regions in which participants are highly involved in the discussion, claims that contents discussed in such a moment are worth putting into summary of discussion [17]. Although clarification of relationship between these moments and active discussion in our case and validation of our insights by a more rigid and generalizable method are needed, our paper contributes on research on teamwork visualization by showing direction of the future research. Our results also show the possibility to detect other team states such as discussion combined with a short individual work, sharing information without discussion or discussion facilitated by one 
member. To distinguish these states, additional audiovisual features need to be considered. Once we can identify team states with some degree of precision, large scale research about its antecedents and consequences would be possible with relatively lower effort than traditional method relying on observing and coding teamwork. In addition, our research can be utilized to develop a tool for supporting teams by displaying team states, which will enhance the performance of transdisciplinary teams. Some assumptions for applying our method are the team size and the length of teamwork. Our method works the best for small teams which have roughly 3 to 7 members and the teamwork for more than 1 hour. Additionally, our result of visualization is sensitive to the quality of an audio data, which is not always guaranteed. We hope our research contributed to the effort for automatic visualization of teamwork.

\section{References}

[1] S. K. Horwitz, and I. B. Horwitz, The Effects of Team Diversity on Team Outcomes: A Meta-Analytic Review of Team Demography, Journal of Management, 2007, Vol. 33, No. 6, pp. 987-1015.

[2] B. N. Waber, D. Olguin Olguin, T. Kim, and A. Pentland, Productivity Through Coffee Breaks: Changing Social Networks by Changing Break Structure, Social Science Research Network, 2010, http://dx.doi.org/10.2139/ssrn.1586375

[3] P. A. Gloor, F. Grippa, J. Putzke, C. Lassenius, H. Fuehres, K. Fischbach and D.Schoder, Measuring social capital in creative teams through sociometric sensors, Int. J. Organisational Design and Engineering, 2012, Vol. 2, No. 4, pp. 380-401.

[4] Y. Takemae, K. Otsuka, and N. Mukawa, An analysis of speakers' gaze behavior for automatic addressee identification in multiparty conversation and its application to video editing, In: 13th IEEE International Workshop on Robot and Human Interactive Communication, Nashville, 2004, pp. 581-586.

[5] L. Chen, R. Travis Rose, Y. Qiao, I. Kimbara, F. Parrill, H. Welji, T.-X. Han, J. Tu, Z. Huang, M. Harper, F. Quek, Y. Xiong, D. McNeill, R. Tuttle, and T. Huang, VACE Multimodal Meeting Corpus, In: Workshop on Machine Learning for Multimodal Interaction, Edinburgh, 2005.

[6] D. Gatica-Perez, L. McCowan, Dong Zhang, and S. Bengio, Detecting group interest-level in meetings, In: IEEE International Conference on Acoustics, Speech, and Signal Processing, Philadelphia, 2005, pp. 489.

[7] R. Vivian, H. Tarmazdi, K. Falkner, N. Falkner, and C. Szabo, The Development of a Dashboard Tool for Visualising Online Teamwork Discussions, In: 37th IEEE International Conference on Software Engineering, Florence, 2015, Vol. 2, pp. 380-388.

[8] S. Basu, T. Choudhury, B. Clarkson, and A. Pentland, Towards measuring human interactions in conversational settings, In: IEEE CVPR Workshop on Cues in Communication, Hawaii, 2001.

[9] J. M. DiMicco, K. J. Hollenbach, A. Pandolfo, and W. Bender, The impact of increased awareness while face-to-face, Human-Computer Interaction, 2007, Vol. 22, No. 1-2, pp. 47-96.

[10] T. Kim, A. Chang, L. Holland, and A. S. Pentland, Meeting mediator: enhancing group collaborationusing sociometric feedback, In: ACM 2008 conference on Computer supported cooperative work - CSCW'08, San Diego, 2008, pp. 457-466.

[11] S. T. Bell, S. G. Brown, A. Colaneri, and N. Outland, Team Composition and the ABCs of Teamwork, American Psychologist, 2018, Vol. 73, No. 4, pp. 349-362.

[12] A. W. Woolley, C. F. Chabris, A. Pentland, N. Hashmi, and T. W. Malone, Evidence for a Collective Intelligence Factor in the Performance of Human Groups, Science, 2010, Vol. 330, No. 6004, pp. 686.

[13] A. Gaggioli, E. Mazzoni, L. Milani, and G. Riva, The creative link: Investigating the relationship between social network indices, creative performance and flow in blended teams, Computers in Human Behavior, 2015, Vol. 42, pp. 157-166.

[14] K. Sawyer, Group genius: The creative power of collaboration, Basic books, New York, 2017.

[15] M. Salanova, A. M. Rodríguez-Sánchez, W. B. Schaufeli, and E. Cifre, Flowing Together: A Longitudinal Study of Collective Efficacy and Collective Flow Among Workgroups, The Journal of Psychology, 2014, Vol. 148, No. 4, pp. 435-455.

[16] S. Culbertson, C. J. Fullagar, M. J. Simmons, and M. Zhu, Contagious Flow: Antecedents and Consequences of Optimal Experience in the Classroom, Journal of Management Education, 2015, Vol. 39, No. 3, pp. 319-349.

[17] C. Lai, J. Carletta, S. Renals, K. Evanini, and K. Zechner, Detecting summarization hot spots in meetings using group level involvement and turn-taking features, In: INTERSPEECH, Lyon, 2013, pp. 2723-2727. 